\title{
Etude de la prévalence des infections à trypanosomes chez différentes espèces d'animaux sauvages du parc national de la Comoé en Côte d'Ivoire : résultats préliminaires sur la comparaison de trois méthodes de diagnostic
}

\author{
C. Komoin-Oka ${ }^{1}$, P. Truc ${ }^{2}$, Z. Bengaly ${ }^{3}$, P. Formenty ${ }^{1}$, G. Duvallet ${ }^{3 *}$, F. Lauginie ${ }^{4}$, J.P. Raath ${ }^{5}$, \\ A.E. N'Depo ${ }^{1}$, Y. Leforban ${ }^{1}$
}

\begin{abstract}
KOMOIN-OKA (C.), TRUC (P.), BENGALY (Z.), FORMENTY (P.), DUVALLET (G.), LAUGINIE (F.), RAATH (J.P.), N'DEPO (A.E.), LEFORBAN (Y.). Etude de la prévalence des infections à trypanosomes chez différentes espèces d'animaux sauvages du parc national de la Comoé en Côte d'Ivoire : résultats préliminaires sur la comparaison de trois méthodes de diagnostic. Revue Élev. Méd. vét. Pays trop., $1994,47(2): 189-194$
\end{abstract}

Si de nombreuses études portent sur la trypanosomose chez les animaux domestiques, peu de recherches unt été effectuées sur la faune sauvage d'Afrique occidentale. Les résultats préliminaires sur la comparaison de trois méthodes de dépistage des trypanosomes : le frottis de sang, la détection des antigènes circulants par la technique ELISA et l'isolement in vitro des trypanosomes par le "kit for in vitro isolation" (KIVI), chez les animaux sauvages du parc national de la Comoé, en Côte d'Ivoire, ont permis de confirmer l'existence de cette infection chez ces animaux sans toutefois identifier de façon précise les espèces de trypanosomes en cause. Des investigations ultérieures permettront d'affiner l'identification des souches de parasites isolées par KIVI.

Mots-clés : Animal sauvage - Trypanosomose - Prévalence - Diagnostic Test ELISA - Prélèvement sanguin - KIVI - Antigène - Côte d'Ivoire.

\section{INTRODUCTION}

En Afrique, les trypanosomoses sont une cause importante de morbidité et de mortalité chez le bétail, dans les zones infestées de glossines. Si de nombreuses études portent sur les animaux domestiques, peu de recherches ont été effectuées sur la faune sauvage, notamment en Afrique occidentale $(2,8,12,14)$. II a donc paru intéressant de profiter du transfert d'un lot d'animaux du parc national de la Comoé, dans une réserve de faune récemment créée, pour effectuer des prélèvements sanguins dans le but de détecter d'éventuelles infections à trypanosomes. Trois méthodes de dépistage ont été utilisées : le frottis de sang, la détection des antigènes circulants par la technique ELISA, et le KIVI, technique d'isolement in vitro des trypanosomes qui a été utilisée avec succès pour le diagnostic parasitologique des trypanosomoses animales et humaines à Trypanosoma brucei $(1,19)$. Les résultats présentés tentent de comparer ces trois méthodes. Cette étude étant toujours en cours de réalisation, les données communiquées, à ce jour, ne sont que des résultats préliminaires.

1. LANADA, Laboratoire de Pathologie animale, BP 206, Bingerville, Côte d'Ivoire.

2. Institut Pierre Richet, OCCGE, BP 1500, Bouaké 01, Côte d'Ivoire.

3. CIRDES/CRTA, 01 BP 454, Bobo-Dioulasso 01, Burkina Faso.

4. Zoo national d'Abidjan, 01 BP 932, Abidjan 01, Côte d'Ivoire.

5. Kruger National Park, PB X402, Skukuza 1350, Afrique du Sud.

${ }^{*}$ Adresse actuelle : CIRAD-EMVT, 10 rue Pierrre Curie, 94704 Maisons-Alfort, Cedex, France.

Reçu le 9.12.1993, accepté le 26.4.1994.

\section{MATÉRIEL ET MÉTHODES}

\section{Région}

Le parc national de la Comoé se situe entre $9^{\circ} 6$ et $8^{\circ} 6$ de latitude nord et entre $4^{\circ} 4$ et $3^{\circ} 1$ de longitude ouest (carte 1 ). II s'étend entre la vallée de la Comoé à l'ouest et celle du Mouhoun à l'est. Les frontières du Burkina Faso et du Ghana se situent à quelques dizaines de $\mathrm{km}$ de ses limites nord et est. II a une superficie de 1150000 ha et son altitude moyenne est de $300 \mathrm{~m}$. II est soumis à un climat tropical subhumide soudanien de transition, mais l'extrême partie nord-est du parc subit l'influence du climat subaride (soudanien) sous la forme d'une nuance climatique plus sèche. Les principales formations végétales sont la forêt claire et la savane, avec quelques îlots de forêt dense et sèche. La faune du parc se caractérise par une grande variété d'espèces, car il se trouve dans une zone de transition où s'interpénètrent les aires de répartition d'espèces animales ouest-africaines de savane et de forêt.

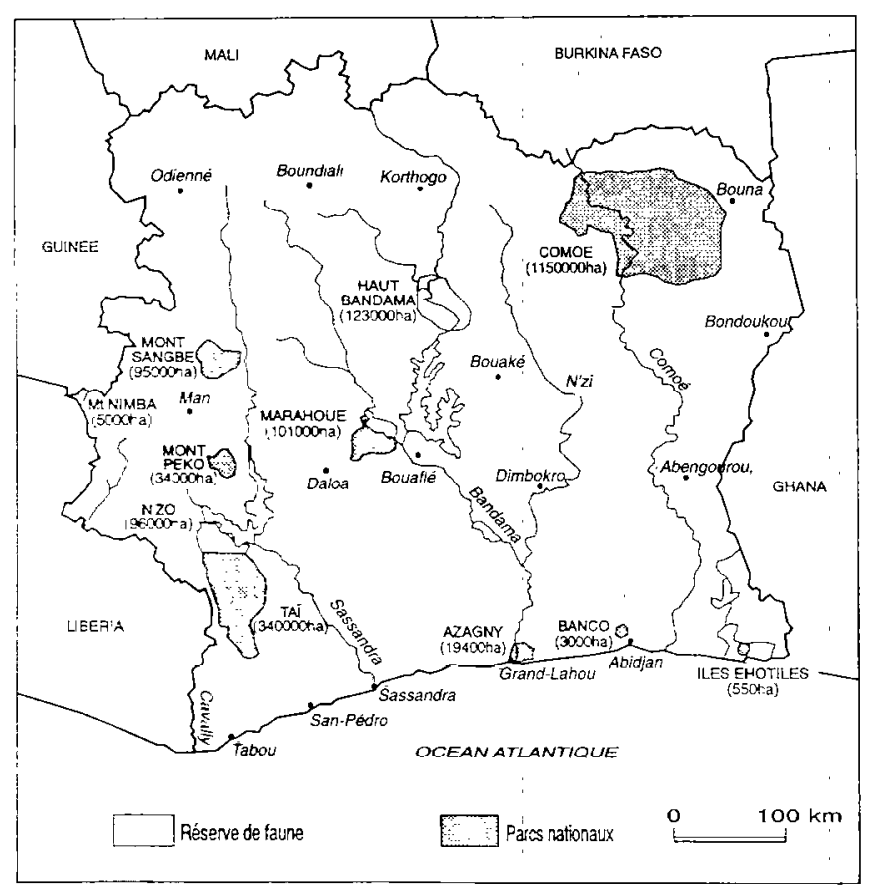

Carte 1: Parcs nationaux et réserves de Côte d'Tloire. 
C. Komoin-Oka P. Truc Z. Bengaly P. Formenty G. Duvallet F. Lauginie J.P. Raath A.E. N'Depo

Y. Leforban

\section{Les prélèvements}

\section{Faune sauvage}

Les prélèvements ont été effectués sur 164 animaux sauvages du parc national de la Comoé. Ces animaux ont été capturés par trois méthodes (téléanesthésie, filets et corrals), puis tranquillisés, soit avec le couple Immobilon/Revivon ${ }^{\circledR}$ (étorphine + acépromazine/diprénorphine), soit avec de l'Haldol ${ }^{\circledR}$ (halopéridol). Ces captures ont été réalisées grâce au concours des parcs nationaux de Côte d'Ivoire, et de l'équipe du parc national Kruger d'Afrique du Sud.

On a ainsi prélevé 61 cobes de Buffon (Kobus kob), 19 cobes Defassa (Kobus defassa), 13 hippotragues (Hippotragus equinus), 53 buffles (Syncerus caffer), 9 bubales (Alcelaphus buselaphus), 6 phacochères (Phacochoerus aethiopicus) et 3 éléphants (Loxodonta africana).

\section{Méthodes de prélèvement}

Les prélèvements ont permis d'effectuer 99 frottis de sang, 160 récoltes de sérum et 96 ensemencements KIVI. Les frottis ont été réalisés par prélèvement à l'oreille, au moment de la capture. Les sérums ont été récoltés après prélèvement de sang sur tube sec à la veine jugulaire des animaux (Venoject ${ }^{\circledR}$ Terumo $^{\circledR}$ ) et centrifugation à 4000 tours $/ \mathrm{min}$ pendant $5 \mathrm{~min}$. Ils ont été conservés à $-20^{\circ} \mathrm{C}$ jusqu'à la réalisation des tests. Les ensemencements KIVI ont été réalisés en prélevant du sang sur anticoagulant (liquoïde 5 p. 100 : polyanétholsulfonate dans une solution saline), et en l'introduisant dans un flacon de milieu GLSH-DCA (comprenant du glucose, de la lactalbumine, du sérum et de l'hémoglobine) (1), selon le protocole modifié par TRUC et al. (19).

\section{Les méthodes de diagnostic}

\section{Le frottis de sang}

Les frottis ont été colorés au laboratoire avec le Giemsa dilué au $1 / 10 e$, après fixation dans de l'alcool méthylique.

\section{Le test de détection des antigènes circulants par ELISA}

On a utilisé le test de détection des antigènes circulants par ELISA, décrit par NANTULYA et LINDQVIST (17). Les anticorps monoclonaux contre Trypanosoma brucei, $T$. congolense et $T$. vivax, ont été gracieusement fournis par l'International Laboratory for Research on Animal Diseases (ILRAD) de Nairobi (Kenya).

Les plaques pour ELISA Maxisorb ${ }^{(B)}$ (Nunc) sont mises à incuber une nuit à $4^{\circ} \mathrm{C}$ avec $100 \mu$ l par puits d'une dilu- tion au 1:500 de chacun des anticorps monoclonaux. Après lavage, les sérums à tester sont déposés sur deux cupules à raison de $100 \mu \mathrm{l}$ de dilution par puits. Pour $T$. brucei, la dilution des sérums est de 1:20; pour $T$. congolense et $T$. vivax, la dilution est de 1:10. Après 15 min d'incubation à température ambiante, les plaques sont rincées et lavées une fois. Ensuite, $100 \mu \mathrm{l}$ du conjugué approprié, dilué au 1:1000 sont déposés par puits. Après 15 min d'incubation à température ambiante, les plaques sont rincées et lavées 3 fois. La solution de substrat (ABTS et eau oxygénée) est ajoutée à raison de $100 \mu \mathrm{l}$ par puits. Les plaques sont lues, après $30 \mathrm{~min}$ d'incubation, sur un lecteur Labsystems Multiskan MCC/340, à une longueur d'onde de $414 \mathrm{~nm}$. A la suite d'essais préliminaires, les seuils de positivité retenus correspondent à une densité optique (DO) de 0,050 pour $T$. brucei et $T$. congolense et une DO de 0,020 pour $T$. vivax.

\section{Le kit pour l'isolement in vitro de trypanosomes (KIVI)}

La trousse d'isolement KIVI a été fournie par l'Institut de Médecine tropicale Prince Léopold d'Anvers, en Belgique. Elle permet de récolter des souches de trypanosomes sanguicoles qui se multiplient, après transformation, sous forme procyclique (1). Un flacon KIVI est ensemencé, par animal. Ces flacons sont transportés au laboratoire et conservés à l'étuve à $27^{\circ} \mathrm{C}$. Après un délai minimal de 3 jours, ils sont contrôlés par étalement d'une goutte de sang/milieu de culture entre lame et lamelle (Gx400) sur 20 champs. Ces contrôles s'effectuent deux fois par semaine durant un mois, puis une seule fois durant le deuxième mois.

Dès qu'un flacon est positif, un repiquage est effectué à raison de $0,1 \mathrm{ml}$ de mélange sang/milieu de culture dans $2 \mathrm{ml}$ de milieu semi-défini (4) supplémenté de 20 p. 100 de sérum de veau fœtal et d'antibiotiques (gentamycine $200 \mu \mathrm{g} / \mathrm{ml}$, pénicilline $5000 \mathrm{Ul} / \mathrm{ml}$ ). Des repiquages successifs sont effectués en vue d'obtenir une concentration suffisante de parasites (supérieure ou égale à antilog. 7.8 , soit $6 \times 10^{7}$ tryps $/ \mathrm{ml}$ ). La culture de masse s'effectue en rajoutant progressivement du milieu, jusqu'à obtention d'un volume total d'environ $250 \mathrm{ml}$. Les culots de parasites sont réalisés par centrifugation à $11000 \mathrm{~g}$, en vue de l'identification taxonomique des souches (dont les résultats seront publiés ultérieurement). En cas de concentration parasitaire inférieure à antilog. 7.8 , une cryocongélation systématique est effectuée afin de préserver la souche qui sera cultivée ou identifiée ultérieurement selon d'autres protocoles.

\section{RÉSULTATS}

Les résultats sont exprimés dans les tableaux I, II et III. Ils permettent la comparaison des trois méthodes de diagnostic et l'évaluation du taux d'infection des différentes espèces animales capturées. 
Résultats combinés des trois méthodes de diagnostic (tableau I)

La présence de trypanosomes a été mise en évidence chez toutes les espèces animales. Bien que les effectifs d'animaux soient très variables selon l'espèce, et parfois très faibles, les résultats obtenus donnent de forts taux d'infection dans l'ensemble, notamment avec les tests KIVI et ELISA-Ag. Les hippotragues, les buffles et les cobes Defassa semblent être les espèces les plus infectées, les pourcentages d'animaux positifs étant élevés pour les trois techniques.

\section{Résultats comparés des trois méthodes de diagnostic (tableau II)}

Les trois méthodes de diagnostic sont comparées en ne tenant compte que des résultats obtenus sur les mêmes animaux. Sur un effectif total de 82 animaux, des trypanosomes ont été mis en évidence chez 20 p. 100 par la méthode du frottis de sang, et chez 80 p. 100 par le test KIVI. L'antigénémie s'est révélée positive sur 50 p. 100 de ces animaux. Les tests KIVI et ELISA-Ag présentent une bonne correspondance dans leurs résultats, d'une façon générale, sauf pour les cobes de Buffon.

\section{Résultats comparés du test de détection des antigènes circulants selon le type d'anticorps monoclonal (tableau III)}

Chez la plupart des espèces animales étudiées, la présence d'antigènes de Trypanosoma brucei, $T$. congolense et $T$. vivax a été révélée. Chez le phacochère, aucun antigène de trypanosomes n'a été mis en évidence.

\section{DISCUSSION}

Les infections à trypanosomes sur des animaux sauvages d'Afrique de l'Ouest ont déjà été décrites $(2,8,14)$ et certains points de l'épidémiologie de la transmission des parasites par les glossines ont été étudiés $(9,11)$. L'utilisation, dans le cadre de cette étude, de trois méthodes de diagnostic de ces infections (le frottis de sang, la détection des antigènes circulants par la technique ELISA et l'isolement in vitro des trypanosomes par le test KIVI) a confirmé la présence de trypanosomes, à des taux d'infection différents, chez les sept espèces d'animaux sauvages capturés dans le parc national de la Comoé. Ces animaux étaient tous apparemment en bonne santé, mais le degré de tolérance à la trypanosomose des mammifères sauvages n'est pas encore clairement établi. En effet, si certains auteurs admettent que les mammifères sauvages infectés et à forte parasitémie peuvent mourir en très peu de temps (13), d'autres, à partir d'infections expérimentales étudiées sur des ongulés sauvages, semblent le contester $(5,10,16,18)$.

L'examen microscopique des frottis de sang a permis de mettre en évidence, chez 17 p. 100 des animaux examinés, et avec une parasitémie très faible, des trypanosomes du type brucei et congolense. Des formes plus massives pourraient correspondre à Trypanosoma vivax, bien qu'il soit difficile d'être catégorique à ce sujet du fait de la qualité des frottis. Les pourcentages obtenus auraient été bien supérieurs si des contraintes, d'ordre pratique, n'avaient empêché d'effectuer une recherche des trypanosomes sanguins par la technique de centrifugation en tube à hématocrite $(15,20)$. Ces données rejoi-

TABLEAU I Résultats combinés des trois méthodes de diagnostic (frottis, KIVI et ELISA-Ag), selon l'espèce animale.

\begin{tabular}{|c|c|c|c|c|c|c|c|c|c|c|}
\hline & \multirow{2}{*}{$\begin{array}{l}\text { Nombre } \\
\text { d'animaux }\end{array}$} & \multicolumn{3}{|c|}{ Frottis } & \multicolumn{3}{|c|}{ KIVI } & \multicolumn{3}{|c|}{ ELISA-Ag } \\
\hline & & $\begin{array}{l}\text { Animaux } \\
\text { examinés }\end{array}$ & $\begin{array}{l}\text { Nombre } \\
\text { de positifs }\end{array}$ & $\begin{array}{c}\text { Pourcentage } \\
\text { de positits }\end{array}$ & $\begin{array}{l}\text { Animaux } \\
\text { examinés }\end{array}$ & $\begin{array}{l}\text { Nombre } \\
\text { de positifs }\end{array}$ & $\begin{array}{l}\text { Pourcentage } \\
\text { de positifs }\end{array}$ & $\begin{array}{l}\text { Animaux } \\
\text { examinés }\end{array}$ & $\begin{array}{l}\text { Nombre } \\
\text { de positifs }\end{array}$ & $\begin{array}{l}\text { Pourcentage } \\
\text { de positifs }\end{array}$ \\
\hline $\begin{array}{l}\text { Eléphant } \\
\text { Loxodonta africana }\end{array}$ & 3 & $\begin{array}{c}\text { Non } \\
\text { fait }\end{array}$ & & & 3 & 0 & 0 & 3 & 2 & 67 \\
\hline $\begin{array}{l}\text { Buffle } \\
\text { Syncerus caffer }\end{array}$ & 53 & 8 & 2 & 25 & 10 & 9 & 90 & 53 & 39 & 74 \\
\hline $\begin{array}{l}\text { Hippotrague } \\
\text { Hippotragus equinus }\end{array}$ & 13 & 9 & 5 & 56 & 9 & 6 & 67 & 13 & 11 & 85 \\
\hline $\begin{array}{l}\text { Bubale } \\
\text { Alcelaphus buselaphus }\end{array}$ & 9 & $\begin{array}{c}\text { Non } \\
\text { fait }\end{array}$ & & & $\begin{array}{c}\text { Non } \\
\text { tait }\end{array}$ & & & 9 & 3 & 33 \\
\hline $\begin{array}{l}\text { Cobe defassa } \\
\text { Kobus defassa }\end{array}$ & 19 & 19 & 5 & 26 & 18 & 17 & 94 & 19 & 15 & 79 \\
\hline $\begin{array}{l}\text { Cobe de buffon } \\
\text { Kobus kob }\end{array}$ & 61 & 57 & 4 & 7 & 53 & 43 & 81 & 60 & 18 & 30 \\
\hline $\begin{array}{l}\text { Phacochère } \\
\text { Phacochoerus aethiopicus }\end{array}$ & 6 & 6 & 1 & 17 & 3 & 1 & 33 & 3 & 0 & 0 \\
\hline TOTAL & 164 & 99 & 17 & 17 & 96 & 76 & 79 & 160 & 88 & 55 \\
\hline
\end{tabular}


C. Komoin-Oka P. Truc Z. Bengaly P. Formenty G. Duvallet F. Lauginie J.P. Raath A.E. N'Depo

Y. Leforban

TABLEAU II Résultats comparés des trois méthodes de diagnostic (frottis, KIVI et ELISA-Ag), selon l'espèce animale.

\begin{tabular}{|c|c|c|c|c|c|c|c|}
\hline & \multirow{2}{*}{$\begin{array}{c}\text { Nombre } \\
\text { d'animaux }\end{array}$} & \multicolumn{2}{|c|}{ Frottis } & \multicolumn{2}{|c|}{ KIVI } & \multicolumn{2}{|c|}{ ELISA-Ag } \\
\hline & & $\begin{array}{l}\text { Nombre de } \\
\text { positifs }\end{array}$ & $\begin{array}{l}\text { Pourcentage de } \\
\text { positifs }\end{array}$ & $\begin{array}{l}\text { Nombre de } \\
\text { positifs }\end{array}$ & $\begin{array}{l}\text { Pourcentage de } \\
\text { positifs. }\end{array}$ & $\begin{array}{l}\text { Nombre de } \\
\text { positifs }\end{array}$ & $\begin{array}{l}\text { Pourcentage de } \\
\text { positifs }\end{array}$ \\
\hline $\begin{array}{l}\text { Buffle } \\
\text { Syncerus caffer }\end{array}$ & 3 & 1 & 33 & 3 & 100 & 3 & 100 \\
\hline $\begin{array}{l}\text { Hippotrague } \\
\text { Hippotragus equinus }\end{array}$ & 9 & 5 & 56 & 6 & 67 & 7 & 78 \\
\hline $\begin{array}{l}\text { Cobe defassa } \\
\text { Kobus defassa }\end{array}$ & 18 & 5 & 28 & 17 & 94 & 15 & 83 \\
\hline $\begin{array}{l}\text { Cobe de buffon } \\
\text { Kobus kob }\end{array}$ & 49 & 4 & 8 & 39 & 80 & 16 & 33 \\
\hline $\begin{array}{l}\text { Phacochère } \\
\text { Phacochoerus aethiopicus }\end{array}$ & 3 & 1 & 33 & 1 & 33 & 0 & 0 \\
\hline TOTAL & 82 & 16 & 20 & 66 & 80 & 41 & 50 \\
\hline
\end{tabular}

TABLEAU III Résultats comparés du test de détection des antigènes circulants, selon le type d'anticorps monoclonal et selon l'espèce animale.

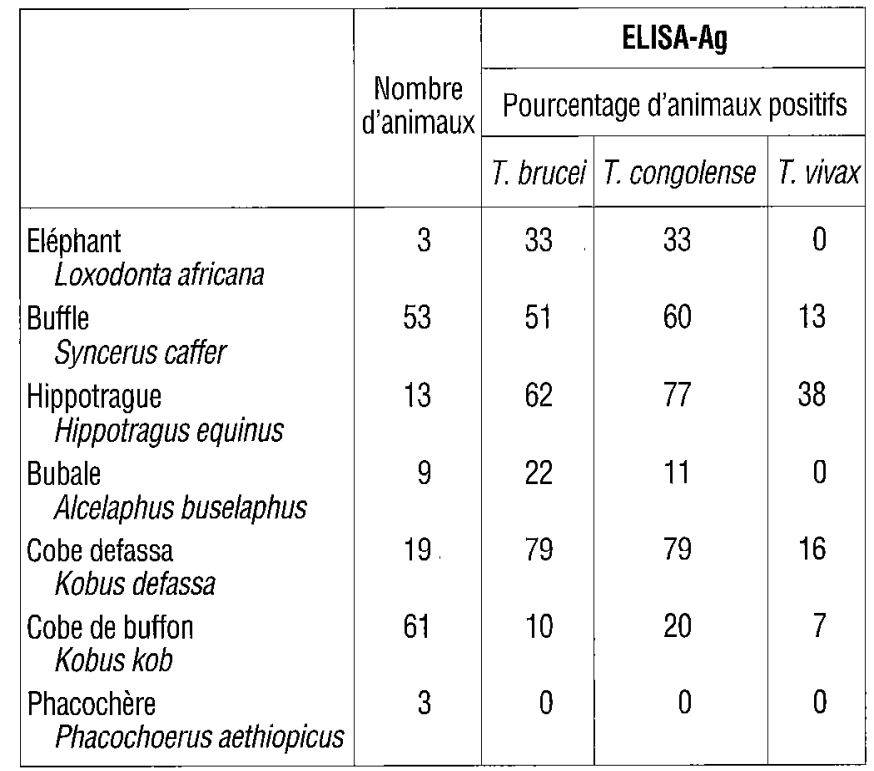

gnent cependant celles obtenues au ranch de gibier de Nazinga, au Burkina Faso, où 15,3 p. 100 des animaux sauvages examinés ont été trouvés positifs à la trypanosomose, et porteurs de trypanosomes des sous-genres Nannomonas, Duttonella, Trypanozoon et Megatrypanum (12). De même, dans la réserve de la biosphère de la Pendjari, au Bénin, la prévalence parasitologique était de 13,92 p. 100, et Trypanosoma congolense, $T$. brucei et $T$. vivax ont été morphologiquement identifiés (6). En Gam- bie, une étude effectuée sur des phacochères a révélé la présence de trypanosomes de l'espèce $T$. simiae (3).

L'isolement in vitro de trypanosomes par le test KIVI a permis l'observation de formes procycliques. Bien que ces formes soient morphologiquement indifférenciables entre les divers groupes, le test a fourni quelques indices:

- l'adaptation rapide de certaines souches suggère la présence de Trypanosoma brucei; en effet, l'obtention rapide des formes grêles en très grand nombre est, à notre connaissance, caractéristique de cette espèce ;

- les faibles concentrations de parasites ou les échecs obtenus pour la culture d'autres souches pourraient s'expliquer par la présence de Trypanosoma congolense, de $T$. vivax, voire de trypanosomes de la section Stercoraria, les milieux de repiquage étant assez sélectifs.

Ces hypothèses semblent être corroborées par les résultats du test de détection des antigènes circulants. Pour la plupart des animaux, ce test a reconnu les parasitémies positives et révélé la présence d'antigènes de Trypanosoma brucei, $T$. congolense et $T$. vivax. Chez le cobe de Buffon, 65: p. 100 des KIVI positifs correspondent à des antigénémies négatives. Les faibles concentrations de parasites et les échecs obtenus pour la culture de certaines de ces souches permettent de penser que les trypanosomes détectés dans ce cas pourraient être des Trypanosoma ingens (dénommés parfois $T$. tragelaphi), appartenant au sous-genre Megatrypanum, des formes de très grande taille ayant été également observées, aussi bien sur le frottis que dans les primo-cultures. Ces grands trypanosomes, non ou très peu pathogènes, ont été décrits chez divers mammifères domestiques et sauvages d'Afrique $(7,12)$. 
Dans l'état actuel de l'avancement des travaux, il est difficile d'identifier, de façon certaine, les espèces des trypanosomes mis en évidence. L'électrophorèse d'isoenzymes en cours permettra d'affiner l'identification taxonomique. Il est également envisagé, en collaboration avec d'autres laboratoires, d'utiliser des sondes spécifiques d'ADN kinétoplastique, ainsi que la technique d'Amplification en Chaîne par Polymérase (ACP). Enfin, la transformation des formes procycliques, par le procédé KIVI, étant parfois incomplète ou insuffisante en vue de l'identification des espèces, un passage cyclique chez la glossine d'élevage, par exemple Glossina morsitans submorsitans et $G$. tachinoides, pourrait être effectué. Après dissection de la glossine, les formes procycliques du tube digestif sont alors plus facilement cultivables.

Des trois méthodes de diagnostic de la trypanosomose, le test KIVI apparaît comme le plus sensible, beaucoup plus que le frottis coloré, un des tests le plus habituellement employé pour le dépistage des trypanosomes. Mais la notion de valeur diagnostique du KIVI est à prendre avec beaucoup de prudence. II s'agit tout au plus d'un diagnostic parasitologique différé (au même titre que l'immunofluorescence ou la détection des antigènes circulants), dont la technicité et le coût n'en font pas un test d'enquête classique, mais qui offre l'avantage d'isoler la souche. Cet isolement ouvre de nombreuses perspectives de recherche. On peut, par exemple, par passage cyclique chez des glossines de laboratoire, obtenir les métacycliques par dissection des glandes salivaires et du proboscis, ce qui présente un intérêt pour l'étude des répertoires antigéniques. La technique ELISA de détection des antigènes circulants est, pour sa part, encore sous évaluation et reste à être validée.

\section{CONCLUSION}

Cette étude de la prévalence des infections à trypanosomes chez différentes espèces d'animaux sauvages du parc national de la Comoé en Côte d'ivoire fournit d'ores et déjà, malgré son caractère préliminaire, des éléments intéressants. La présence de trypanosomes a été mise en évidence chez les 7 espèces de mammifères sauvages. Divers indices font suspecter leur appartenance aux espèces Trypanosoma brucei, $T$. congolense et $T$. vivax; il est possible que des trypanosomes de la section Stercoraria soient également présents.

L'utilisation des trois méthodes de diagnostic (le frottis de sang, la détection des antigènes circulants par l'ELISA et l'isolement in vitro des trypanosomes par le KIVI) n'a pas permis l'identification fine des trypanosomes en cause, mais elle ouvre de nombreuses perspectives en matière de recherche et montre l'intérêt des nouvelles techniques de diagnostic que sont l'ELISA et le KIVI. Des investigations, en cours actuellement, permettront d'affiner l'identification taxonomique des souches de trypanosomes isolées.

\section{REMERCIEMENTS}

Nous tenons à manifester notre gratitude au personnel des parcs nationaux de Côte d'Ivoire, ainsi qu'à l'équipe du parc national Kruger d'Afrique du Sud qui ont réalisé les captures des animaux. Nous remercions également le Docteur FANNY N'GOLO, pour ses précieuses informations relatives au parc national de la Comoé, et MM. N'GUESSAN Paul, SADIA Mamadou et ASSI Alfred, pour leur collaboration technique au laboratoire.

\section{BIBLIOGRAPHIE}

1. AERST (D.), TRUC (P.), PENCHENIER (L.), CLAES (Y.), LE RAY (D.). A kit for in vitro isolation of trypanosomes in the field : first trial with sleeping sickness patients in the Congo Republic. Trans. R. Soc. trop. Med. Hyg., 1992, 86 : 394-395.

2. Annual report, 1984. Monrovia, Liberia Research Unit of the Tropical Institute Hamburg. 1985

3. CLAXTON (J.R.), FAYE (J.A.), RAWLINGS (P.). Trypanosome infec tions in warthogs (Phacochoerus aethiopicus in The Gambia. Vet. Parasitol., 1992, 41 (3-4) : 179-187

4. CUNNINGHAM (I.). New culture medium for maintenance of Tsetse tissues and growth of Trypanosomatids. J. Protozool., 1977, 21 (2): 325-329.

5. GROOTENHUIS (J.G.), VARMA (Y.), BACK (S.), MOLOO (S.K.), AKUL (G.W.O.), EMERY (D.L.), MURKAY (M.). Host response of some African wild Bovidae to experimental trypanosome infection. In : KARBE (E.), FREITAS (E.K.), eds. Trypanotolerance and animal production. Proc. Workshop, Lomé, Togo, 10-14 May 1982, Eschborn, GTZ, 1982. p. 37-42 $\left(n^{\circ} 16\right)$.

6. GUEDEGBE (B.), VERHULST (A.), VAN MEIRVENNE (N.), PAN DEY (V.S.), DOKO (A.). Indications sérologiques de l'existence d'un réservoir sauvage du Trypanosoma brucei gambiense dans la réserve de la biusphère de la Pendjari en République du Bénin. Annls Soc. belge Méd. trop., 1992, 72 (2) : 113-120.

7. ITARD (J.). Les trypanosomoses animales africaines. In : Précis de parasitologie vétérinaire tropicale. Paris. Ministère de la Coopération et du Développement, 198I. p. 303-469 (Manuels et précis d'élevage, $\mathrm{n}^{\circ} 10$ )

8. KUPPER (W.), WOLTERS (M.), TSCHARF (I.). Observations on kob antelope (Kobus kob) in Northern Ivory Coast and their epizootiological role in trypanosomiasis transmission. Z. angew Zool., 1983, 70 : 227-283.

9. LAVEISSSIERE (C.). BOREHAM (P.F.L.). Ecologie de Glossina tachinoides Westwood, 1850 . en savane humide d'Afrique de l'Ouest. I Préférences trophiques. Cah. ORSTOM, Sér. Ent. méd. Parasit., 1976, 14 (3) : 187-200.

10. MAKLMYAVIRI (A.M.). L’importance épidémiologique de l'hôte expérimental et du réservoir animal de Trypanosoma brucei gambiense. Revule Méd. vét., 1990. 141 (11) : 873-875.

11. MATTIOLI (R.C.). Fréquence des trypanosomes dans les populations de glossines du ranch de gibier de Nazinga (Burkina Faso). Revue Elev. Méd. vét. Pays trop.. 1991, 44 (2) : 165-168.

12. MATTIOLI (R.C) . JEAN (O.). BELEM (A.M.G.). Incidence de la trypanosomose sur la faune sauvage d'un ranch de gibier au Burkina Faso. Revue Elev. Méd. vét. Pays trop., 1990, 43 (4) : 459-465.

13. MEHLITZ (D.) Trypanosomes in African wild animals. In: BAKER (J.R.). Perspectives in try'panosomiasis research. Chichester, Angleterre, Research Studies Press, 1982, p. 25-35. 


\section{Komoin-Oka P. Truc Z. Bengaly P. Formenty G. Duvallet F. Lauginie J.P. Raath A.E. N'Depo Y. Leforban}

14. MEHLITZ (D.). Le réservoir animal de la maladie du sommeil à Trypanosoma brucei gambiense. Eschborn, GTZ/Maisons-Alfort, IEMVT, 1986. $156 \mathrm{p}$. (Etudes et synthèses de l'IEMVT n ${ }^{\circ} 18$ )

15. MURRAY (M.), MURRAY (P.K.), McINTYRE (W.I.M.). An improved parasitological technique for the diagnosis of African trypanosomiasis. Trans. R. Soc. trop. Med. Hyg., 1977, 71:325-326.

16. MURRAY (M.), GROOTENHUIS (J.G.), AKOL (G.W.O.), EMERY (D.L.), SHAPIRO (S.Z.), MOLOO (S.K.), FAIQA DAR, BOVELL (D.L.), PARIS (J.). Potential application of research on African trypanosomiases in wildlife and preliminary studies on animals exposed to tsetse infected with Trypanosoma congolense. In: KARSTAD (L.), NESTEL (B.), GRAHAM (M.), eds. Wildlife disease research and economic development. Proc. of a workshop held in Kabete, Kenya, 8-9 September 1980. Ottawa, IDRC, 1981. p. $40-45$.

KOMOIN-OKA (C.), TRUC (P.), BENGALY (Z.), FORMENTY (P.), DUVALLET (G.), LAUGINIE (F.), RAATH (J.P.), N'DEPO (A.E.), LEFORBAN (Y.). Trypanosome infections and wild animals in Comoe national park (Côte d'Ivoire) : Preliminary results on the comparison of three methods of diagnosis. Revue Élev. Méd. vét. Pays trop., $1994,47(2)$ : $189-194$

Compared with the numerous studies of trypanosomosis in domestic animals, few such studies have been carried out on wild animals in West Africa. Preliminary results on the comparison of three detection methods (thin smears, detection of trypanosome antigens by ELISA-Test and Kit for in vitro isolation of trypanosomes, KIVI) in wild animals of Comoe Game Reserve in Côte d'Ivoire confirm the actual presence of trypanosomes; however, no accurate identification of those parasites has been possible, but work is in progress to clarify the taxonomical status of stocks isolated by KIVI.

Key words : Wild animal - Trypanosomosis - Prevalence - Diagnosis ELISA test - Blood sampling - KIVI - Antigen - Côte d'Ivoire.
17. NANTULYA (V.M.), LINDQVIST (K.J.). Antigen detection enzyme immunoassays for the diagnosis of Trypanosoma vivax, $T$. congolense and T. brucei infections in cattle. Trop. Med. Parasit., 1989, 40 : 267-272

18. OLUBAYO (R.). Trypanosomiasis of game animals. In: LOSOS (G.), CHOUINARD (A.), eds. Pathogenicity of trypanosomes. Proc. workshop, Nairobi, Kenya, 20-23 Nov. 1978 . Ottawa, IDRC, 1979, p. 87-88.

19. TRUC (P.), AERTS (D.), MC NAMARA (J.J.), CLAES (Y.), ALLINGHAM (R.), LE RAY (D.), GODFREY (D.G.). Direct isolation in vitro of Trypanosoma brucei from man and other animals, and its potential value for the diagnosis of gambian trypanosomiasis. Trans. R. Soc. trop. Med. Hyg., 1992, 86: 627-629.

20. VERY (P), BOCQUENTIN (R.), DUVALLET (G.). Sensibilité de la double microcentrifugation pour la recherche des trypanosomes.' Revue Elev. Méd. vét. Pays trop., 1990, 43 (3) : 325-329.

KOMOIN-OKA (C.), TRUC (P.), BENGALY (Z.), FORMENTY (P.), DUVALLET (G.), LAUGINIE (F.), RAATH (J.P.), N'DEPO (A.E.), LEFURBAN (Y.). Estudio sobre la prevalencia de infecciones por tripanosomas en diferentes especies de reses bravías del Parque Nacional de Comoe, Costa de Marfil: resultados preliminares sobre la comparación de tres métodos diagnósticos. Revue Élev. Méd. vét. Pays trop., 1994, 47 (2) : 189-194

A pesar de la gran cantidad de estudios realizados sobre la tripanosomosis en los animales domésticos, muy pocos estudios se han llevado a cabo sobre la fauna bravía de Africa occidental. Los resultados preliminares sobre la comparación de tres métodos' diagnósticos de detección de tripanosomas (frotis sanguíneo, detección de antígenos circulantes mediante el ELISA y aislamiento in vitro de los tripanosomas por el KIVI (Kit de aislamiento in vitro del tripanosoma)), en la fauna bravía del Parque Nacional de Comoe, Costa de Marfil, han permitido la confirmación de la existencia de esta infección en estos animales, sin por eso permitir la identificación precisa de las especies y cepas de parásitos aislados por KIVI. Se necesitaran investigaciones ulteriores.

Palabras clave : Res bravía - Tripanosomosis - Prevalencia Diagnóstico - Test ELISA - Muestro sanguineo - KIVI - Antígeno Costa de Marfil. 